\title{
Striatal and Nigral Neuron Subpopulations in Rigid Huntington's Disease: Implications for the Functional Anatomy of Chorea and Rigidity-Akinesia
}

\author{
Roger L. Albin, MD,* Anton Reiner, PhD, † Keith D. Anderson, PhD, † John B. Penney, MD,* \\ and Anne B. Young, MD, PhD*
}

\begin{abstract}
Neuropeptide immunohistochemistry was used to test several hypotheses of the anatomical bases of chorea and rigidity-akinesia. To test the hypothesis that elevated concentration of striatal somatostatin causes chorea, we visually compared the density of striatal neurons containing somatostatin and neuropeptide $\mathbf{Y}$ in brains affected by choreic or rigid-akinetic Huntington's disease (HD). The density of these neurons was elevated in both rigid-akinetic and choreic HD specimens with an apparently normal total number of these neurons, indicating that elevated somatostatin concentration, by itself, does not lead to chorea. We tested the hypothesis that rigid-akinetic HD results from deficient dopaminergic nigrostriatal neurotransmission by examining tyrosine hydroxylase-immunoreactive (TH-IR) neurons in the substantia nigra. In rigid-akinetic HD brains, there was no obvious reduction of nigral TH-IR neurons, indicating that rigid-akinetic HD is probably not due to loss of nigral dopaminergic neurons. Finally, we also examined the status of striatal projection neurons and found near total loss of all striatal neurons projecting to the lateral globus pallidus, medial globus pallidus, and substantia nigra in brains affected by rigid-akinetic HD in contrast to the preservation of neurons projecting to the medial globus pallidus in choreic HD. These results are consistent with the hypothesis that chorea results from preferential loss of striatal neurons projecting to the lateral globus pallidus and that rigid-akinetic HD is a consequence of the additional loss of striatal neurons projecting to the medial segment of the pallidum.
\end{abstract}

Albin RL, Reiner A, Anderson KD, Penney JB, Young AB. Striatal and nigral neuron subpopulations in rigid Huntington's disease: implications for the functional anatomy of chorea and rigidity-akinesia. Ann Neurol 1990;27:357-365

An understanding of the mechanisms underlying chorea has long eluded neurologists. While chorea is associated with striatal pathology, efforts to produce chorea in animals by creating striatal lesions have been unsuccessful [1-3]. Similarly, traumatic or vascular lesions of the striatum in humans do not generally cause chorea $[4,5]$. This apparent paradox has led to speculation that chorea may result from the preferential loss of specific subpopulations of striatal neurons, with selective preservation of other striatal neuron subpopulations $[6,7]$.

Along these lines, one recent hypothesis regarding chorea is that it results from a relative excess of somatostatin (SS) in the striatum [6]. This idea derives from the observation that striatal interneurons containing both SS and neuropeptide $Y$ (NPY) are spared in Huntington's disease (HD) $[8,9]$. The resulting rela- tive increase in the concentration of striatal SS is thought to influence the action of dopamine in the striatum. SS enhances dopamine release by nigrostriatal terminals and may potentiate the effect of dopamine on striatal neurons [10-12]. Intrastriatal injection of SS agonists in rats produces stereotypical behavior similar to that seen with the administration of dopamine agonists [12].

An alternative hypothesis is that chorea results from selective degeneration of striatal neurons projecting to the external globus pallidus (GPe) with preservation of neurons projecting to the internal globus pallidus (GPi) $[1,7]$. This hypothesis is supported by the recent discovery of preferential loss of striatal neurons projecting to the GPe in the early stages of HD [13] and by the development of a nonhuman primate model of chorea in which hyperkinetic dyskinesias are
From the *Department of Neurology, University of Michigan, Ann Arbor, MI, and the †Department of Anatomy and Neurobiology, University of Tennessee Health Science Center, Memphis, TN.
Received Jul 10, 1989, and in revised form Sep 21. Accepted for publication Sep 22, 1989.

Address correspondence to Dr Albin, Neuroscience Laboratory Building, 1103 E. Huron, Ann Arbor, MI 48104. 
Table 1. Clinical Characteristics and Postmortem Specimen Characteristics of Rigid HD Cases

\begin{tabular}{|c|c|c|c|c|c|c|c|}
\hline Case No. & Sex & $\begin{array}{l}\text { Age at } \\
\text { Death }(y r)\end{array}$ & Grade & $\begin{array}{l}\text { Postmortem } \\
\text { Delay (hr) }\end{array}$ & $\begin{array}{l}\text { Fixation } \\
\text { Time }\end{array}$ & Symptoms & Medications \\
\hline 1 & $\mathrm{~F}$ & 31 & 4 & 24 & $24 \mathrm{mo}$ & $\begin{array}{l}\text { Rigidity, bradykinesia, } \\
\text { juvenile-onset }\end{array}$ & Carbidopa-levodopa \\
\hline 2 & $\mathrm{~F}$ & 11 & 4 & 9 & 3 wk & $\begin{array}{l}\text { Rigidity, bradykinesia, } \\
\text { spasticity, dementia, } \\
\text { epilepsy, juvenile-onset }\end{array}$ & $\begin{array}{l}\text { Phenytoin, valproate, } \\
\text { baclofen, phenobarbital, } \\
\text { methadone, clonazepam }\end{array}$ \\
\hline 3 & $\mathbf{F}$ & 20 & 4 & 18 & $16 \mathrm{mo}$ & $\begin{array}{l}\text { Dementia, rigidity, } \\
\text { epilepsy, juvenile-onset }\end{array}$ & $\begin{array}{l}\text { Diazepam, haloperidol, } \\
\text { phenytoin }\end{array}$ \\
\hline 4 & $\mathrm{~F}$ & 34 & 3 & $?$ & $6 \mathrm{mo}$ & Rigidity, adult-onset & $\begin{array}{l}\text { Chlorpromazine, } \\
\text { thioridazine, naproxen }\end{array}$ \\
\hline Mean & & 24 & & 17 & $12 \mathrm{mo}$ & & \\
\hline
\end{tabular}

induced by pharmacological blockade of striatal input to the GPe [14].

To evaluate these hypotheses we used immunohistochemistry to determine the status of striatal interneurons containing SS and NPY and striatal neuropeptide-containing projection neurons in 4 cases of rigidakinetic $H D$. This unusual variant of $H D$, in which the presentation and clinical course are dominated by rigidity and bradykinesia rather than chorea, offers an opportunity to test theories of chorea. If SS excess is the sole cause of chorea, then one would expect that brains affected by rigid-akinetic HD, which lack chorea, should not exhibit the sparing of striatal neurons containing SS and NPY seen in HD. Conversely, if selective degeneration of striatal neurons projecting to the GPe with sparing of those projecting to the $G P i$ is the cause of chorea, then one would predict that specimens from patients with rigid HD should not exhibit the selective loss of striatal neurons projecting to the GPe and should exhibit a different pattern of loss of striatal projection neurons.

In addition, we evaluated also the status of striatal neurons projecting to the substantia nigra (SN) and tyrosine hydroxylase-containing neurons in the SN. The latter are of particular interest because Stoessel and colleagues suggested that the rigidity sometimes seen in HD is a result of deficient nigrostriatal transmission [15].

\section{Methods}

\section{Patient Material}

We studied specimens from 4 cases of rigid-akinetic HD (Table 1). Three of the 4 cases had onset before the age of 20 (juvenile onset), and 1 had onset in the early twenties (early adult onset). All had a clinical course dominated by rigidity and bradykinesia with little or no chorea (see Table 1 for clinical characteristics). All specimens were pathologically graded according to the criteria of Vonsattel and associates [16]. All specimens from cases of juvenile-onset HD were grade 4 , while the specimen from the one with adult-onset HD was grade 3. One specimen was obtained from the Pathology Department at the University of Michigan Medical Center, 1 from the Pathology Department of Stanford University, and 2 from the Brain Tissue Resources Center (BTRC; Belmont, MA). Brains were obtained at autopsy and were fixed in either unbuffered 15\% formalin (University of Michigan and Stanford University) or $10 \%$ neutral buffered formalin (BTRC). The average postmortem delay prior to fixation was 17 hours. After 2 to 3 weeks of fixation, the brains were blocked and stored in 10 to $15 \%$ formalin $0.1 \mathrm{M}$ phosphate buffer ( $\mathrm{PB} ; \mathrm{pH} 7.4$ ). The average duration of fixation was 12 months. We also examined specimens from a previously described group of patients with choreic $\mathrm{HD}$ and previously described neurologically normal and abnormal control subjects [13]. Both the rigid-akinetic and choreic specimens described here were used in our previous study of striatal projection neurons in HD [13] but extensive clinicopathological correlations were not attempted in that study. For comparison with the cases of rigid HD we used 4 specimens of well-documented choreic HD. The average age at death of the patients who had choreic HD was 49 years (range, 19-81 years); the average postmortem delay was 12.5 hours (range, 2-24 hours); the average duration of fixation was 12.1 months (range, 0.5-18 months); and the specimens were grades 2 or 3 . Of the choreic group 1 had juvenile-onset HD (age 19 at death) with grade 3 pathology. For control specimens (total number $=13$ ), the average age at death was 56 years (range, 10-81 years); the average postmortem delay was 13 hours (range, 5-26 hours); and the average duration of fixation was 9.6 months (range, 0-21 months). The controls included specimens from children and young adults (ages 10,15,27, and 34 years). The results from specimens in these younger subjects were identical to those obtained in older adults.

\section{Immunobistochemistry}

Tissue blocks containing putamen and pallidum or SN were recovered from formalin and immersed in $20 \%$ sucrose $10 \%$ glycerol PB containing $0.01 \%$ sodium azide at $4^{\circ} \mathrm{C}$ until the time of assay. Forty-micron-thick frozen sections were cut on a sliding microtome and immunohistochemistry was per- 
formed using the peroxidase-antiperoxidase technique as previously described [13]. To examine striatal interneurons containing SS and NPY we used several well-characterized and highly specific primary antisera directed against SS (INCstar Inc, Stillwater, MN; dilution 1:500; and gift of Drs S. Vincent and J. Brown, dilution 1:500) [17] and NPY (gift of Dr J. Polak, dilution 1:500; and gift of Drs J. R. Oliver and W. Blessing, dilution 1:500 [18, 19]. The integrity of striatal projection neurons was assessed by visualization of enkephalin-immunoreactive nerve terminals in the GPe, and substance P-immunoreactive nerve terminals in the GPi and SN. Changes in the abundance of labeled terminals were used to infer changes in the density of striatal neurons projecting to the GPe, GPi, and SN. We used wellcharacterized antisera directed against substance $P$ (Accurate Chemical and Scientific, Westbury, NY; dilution $1: 2,000$ ), [Leu]-enkephalin (INCstar, dilution $1: 1,000$ ) and [Met]enkephalin-Arg ${ }^{6}-\mathrm{Gly}^{7}-\mathrm{Leu}^{8}$ (gift of Dr G. J. Dockray, dilution 1:500). The latter two antisera both stain enkephalinergic nerve terminals and produce identical staining patterns [13]. Dopaminergic nigral neurons and neuropil were visualized using a highly specific antiserum directed against tyrosine hydroxylase (Eugenetech, Allandale, NJ; dilution 1:4,000). Sections were viewed under bright field illumination and the changes in neuropeptide-containing perikarya and nerve terminals and tyrosine hydroxylasecontaining perikarya were qualitatively assessed by comparison with control and choreic HD specimens. In 1 case of juvenile-onset rigid HD (Case 1), a pediatric control case (age 10), and a case of juvenile-onset choreic HD, sections were taken at equivalent levels of the putaminopallidal complex and the distribution of striatal neurons containing SS and NPY was charted using a camera lucida technique.

\section{Results}

\section{Striatal Interneurons Containing SS and NPY}

The striatal neurons containing SS and NPY were readily visualized in all specimens (Fig 1). As with choreic HD specimens, the areal density of these interneurons in the atrophied putamen of all rigid specimens appeared elevated compared with control specimens (Table 2). Charting and subsequent counting of neurons containing SS and NPY in 1 case of rigid HD, 1 choreic case, and a pediatric control case revealed no evident difference in the number of NPY-immunoreactive neurons between these specimens (Fig 2).

\section{Peptidergic Striatal Projection Neuron Terminals}

In the control specimens, there was robust staining of enkephalinergic terminals in the GPe and substance $\mathrm{P}$-containing terminals in the GPi and $\mathrm{SN}$ (see Table 2, Figs 3 and 4). In all grade 4 rigid cases there was near total depletion of peptidergic terminals in the $\mathrm{GPe}, \mathrm{GPi}$, and SN, indicating near total depletion of the striatal projection neurons giving rise to these terminals. In the one grade 3 rigid-akinetic specimen, there was substantial loss of enkephalin immunoreactivity in the GPe and a somewhat lesser loss of sub- stance $\mathrm{P}$ immunoreactivity in the GPi (see Table 2). Substance $\mathrm{P}$ immunoreactivity in $\mathrm{SN}$ was markedly depleted in this specimen. In the grade 3 , juvenileonset choreic specimen and in other choreic specimens, there was substantial sparing of substance $P$ immunoreactivity in the GPi and great loss of enkephalin immunoreactivity in the GPe (see Fig 4).

\section{Tyrosine Hydroxylase Immunoreactivity in the $S N$}

In all specimens there was a normal distribution and abundance of tyrosine hydroxylase-immunoreactive neuropils and neurons in the SN (Fig 5).

\section{Discussion}

We used immunohistochemistry to evaluate the status of striatal and nigral neuron subpopulations in rigid HD. Striatal interneurons containing SS and NPY and nigral neurons containing tyrosine hydroxylase were directly visualized, and the density of striatal projection neurons was indirectly assessed by visualization of neuropeptide-containing terminals in the GPe, GPi, and SN. This latter approach is necessary because it is difficult to visualize the perikarya of striatal projection neurons without intraventricular colchicine treatment, a technique not applicable to humans. The majority of striatal projection neurons send their efferents to only one target region [20-22], and can also be differentiated by which neuropeptide they contain. Striatal neurons projecting to the GPe contain enkephalins, while striatal neurons projecting to the $\mathrm{GPi}$ and $\mathrm{SN}$ contain substance $P[23,24]$. Consequently, it is possible to examine the density of neuropeptide-immunoreactive terminals in the $\mathrm{GPe}, \mathrm{GPi}$, and $\mathrm{SN}$ and make reliable inferences about the integrity of subpopulations of striatal projection neurons giving rise to those terminals. We assume that loss of immunoreactivity in the terminal fields is the result of striatal neuron degeneration but we cannot exclude the possibility that loss of neuropeptide immunoreactivity in the terminal fields represents dysfunction of striatal neurons rather than degeneration.

Our results are inconsistent with the hypothesis that elevated striatal concentrations of SS are the sole cause of chorea. In all specimens of rigid HD, we found apparently complete preservation of striatal interneurons containing SS and NPY, indicating that another mechanism is responsible for chorea. This conclusion is supported by the results of a clinical trial in which cysteamine, an agent known to deplete central nervous system SS, was administered to patients with choreic HD [25]. No amelioration of chorea was seen. Experimental data also support the conclusion that elevated striatal concentration of SS is not the cause of chorea. Leblanc and associates recently reported the results of intrathecal infusion of SS agonists in nonhu- 

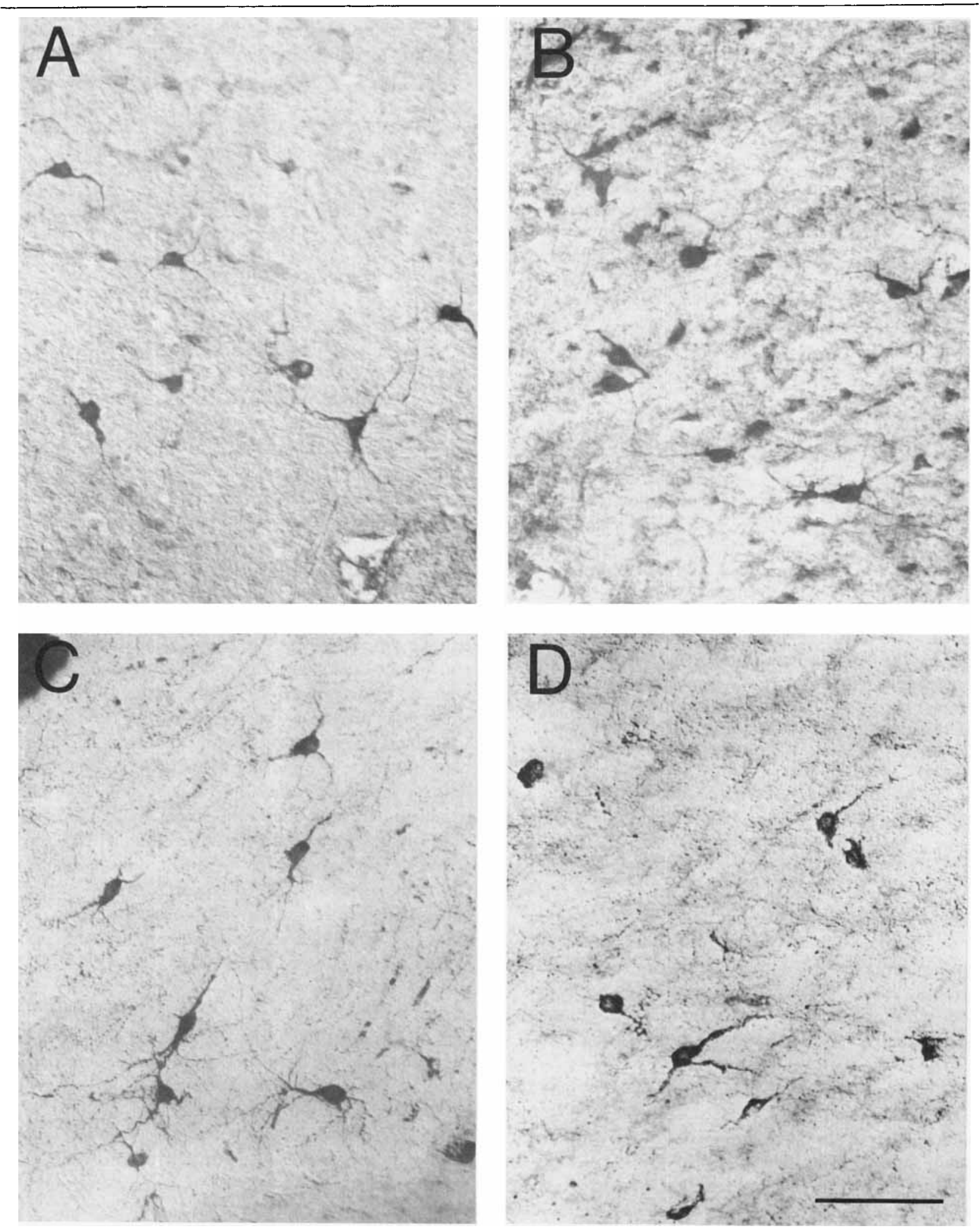

Fig 1. Striatal neurons immunohistochemically labeled for neuropeptide $Y$ (NPY). (A) The putamen of a normal juvenile. (B) The putamen from a case of choreic juvenile-onset $H D$ (grade 3). (C) The putamen from a case of rigid adult-onset $H D$ (Case 4, grade 3). (D) The caudate from a case of rigid juvenile-onset $H D$ (Case 3, grade 4). Scale bar $=100 \mu \mathrm{m}$. 
Table 2. Summary of Immunobistochemical Findings in Rigid HD Specimens

\begin{tabular}{|c|c|c|c|c|c|c|}
\hline Case No. & Put SS-IR & Put NPY-IR & GPe ENK-IR & GPi SP-IR & SN SP-IR & SN TH-IR \\
\hline 1 & $\uparrow \uparrow$ & $\uparrow \uparrow$ & $\downarrow \downarrow \downarrow$ & $\downarrow \downarrow \downarrow$ & $\downarrow \downarrow \downarrow$ & Normal \\
\hline 2 & $\uparrow \uparrow$ & $\uparrow \uparrow$ & $\downarrow \downarrow \downarrow$ & $\downarrow \downarrow \downarrow$ & $\downarrow \downarrow \downarrow$ & Normal \\
\hline 3 & $\uparrow \uparrow$ & $\uparrow \uparrow$ & $\downarrow \downarrow \downarrow$ & $\downarrow \downarrow \downarrow$ & $\downarrow \downarrow \downarrow$ & Normal \\
\hline 4 & $\uparrow \uparrow$ & $\uparrow \uparrow$ & $\downarrow \downarrow \downarrow$ & $\downarrow \downarrow$ & $\downarrow \downarrow$ & Normal \\
\hline
\end{tabular}

Put $=$ putamen; $\mathrm{GPe}=$ external globus pallidus; GPi $=$ internal globus pallidus; $\mathrm{SN}=$ substantia nigra; SS-IR $=$ somatostatinimmunoreactive; NPY-IR = neuropeptide Y-immunoreactive; ENK-IR = enkephalin-immunoreactive; SP-IR = substance Pimmunoreactive; TH-IR = tyrosine hydroxylase-immunoreactive; $\uparrow \uparrow=$ markedly increased density of immunoreactive perikarya; $\downarrow \downarrow \downarrow=$ near total depletion of immunoreactive terminals; $\downarrow \downarrow=$ markedly decreased density of immunoreactive terminals.

man primates [26]. They observed bradykinesia in animals receiving chronic infusion of the long-acting SS agonist SMS 201-995, which indicates that in primates in vivo, the SS agonist elicits effects similar to those induced by dopamine antagonists. Consistent with this hypothesis, the bradykinesia was reversed by administration of the dopamine agonist apomorphine. The results of Leblanc and associates directly contradict the idea that SS potentiates the effect of dopamine in the striatum in primates and that chorea results from excess striatal SS. Their results are also inconsistent with data obtained in rodents and suggest the existence of an interspecies difference between primates and rats in the effect of SS in the striatum.

Our results are consistent with the hypothesis that chorea results from selective loss of striatal neurons projecting to the GPe. In a previous study, we found that grade 2 and some grade $3 \mathrm{HD}$ specimens are marked by selective loss of neurons projecting to the $\mathrm{GPe}$. In some of the cases studied in that report there was enough clinical information available to correlate the selective loss of neurons projecting to the GPe with the presence of chorea [13]. In the rigid-akinetic grade 3 and 4 specimens reported in the present study, we found evidence of profound loss of striatal neurons projecting to the GPe, GPi, and SN. This nonselective loss of striatal projection neurons correlates with the rigidity and lack of chorea exhibited by the patients. Our analysis of these rigid-akinetic cases does not, however, constitute a definitive test of the hypothesis that chorea results from selective loss of striatal neurons projecting to the $\mathrm{GPe}$, because we were able to examine only specimens from cases with grade 3 and 4 rigid HD. It is still possible that selective loss of striatal projections to the GPe might be observed in earlier grades of rigid-akinetic HD. A better test of the hypothesis would be to evaluate cases of rigid-akinetic HD at earlier grades of HD and to compare earlygrade rigid $\mathrm{HD}$ with early-grade choreic $\mathrm{HD}$.

The hypothesis that chorea results from selective loss of striatal neurons projecting to the GPe and that rigidity-akinesia is associated with loss of striatal neurons projecting to both the GPe and GPi implies that

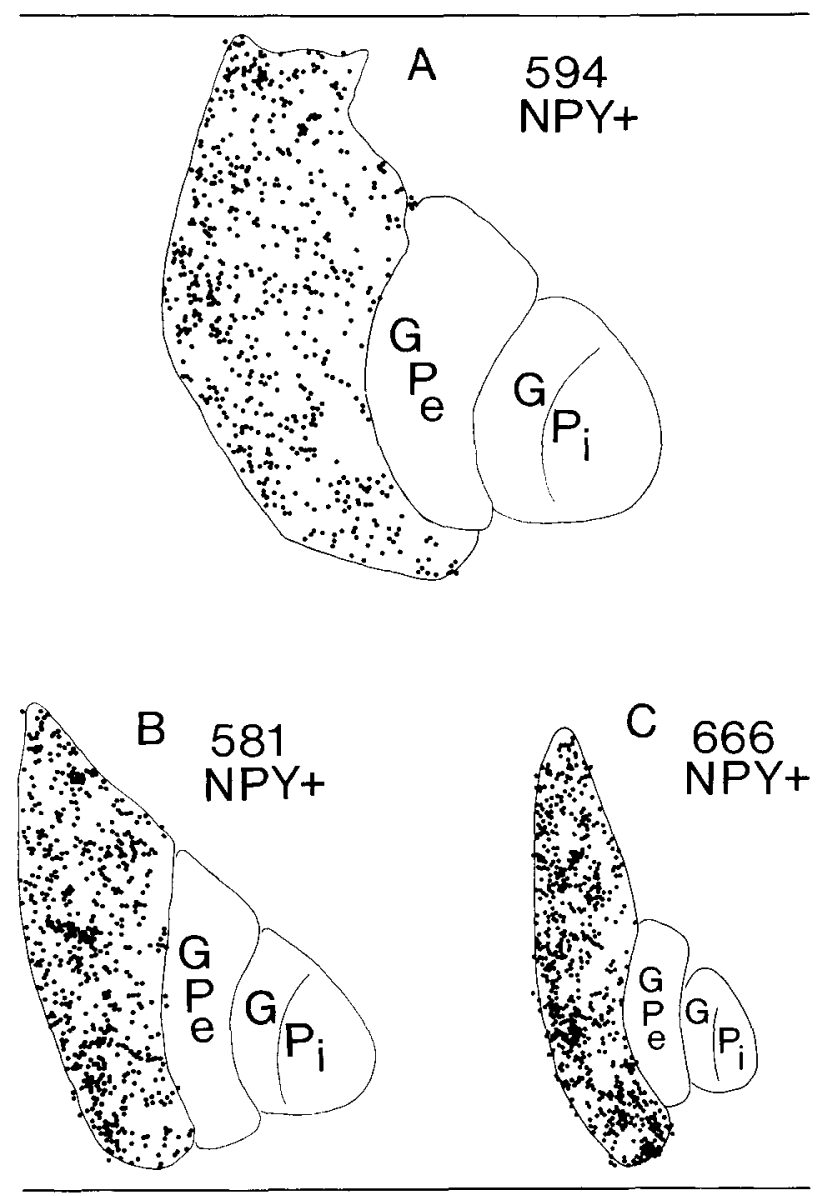

Fig 2. Camera lucida reconstructions of the distributions of neuropeptide $Y$-immunoreactive $(N P Y+)$ neurons at comparable levels of the basal ganglia. (A) A normal juvenile. (B) Choreic juvenile-onset $H D$ (grade 3). (C) Rigid juvenileonset $H D$ (Case 3, grade 4). Although the number of $N P Y$ immunoreactive perikarya is similar, shrinkage of the putamen greatly elevates the packing density of these neurons in grade 3 and $4 \mathrm{HD} . \mathrm{GPe}=$ external globus pallidus; $G P i=$ internal globus pallidus. 


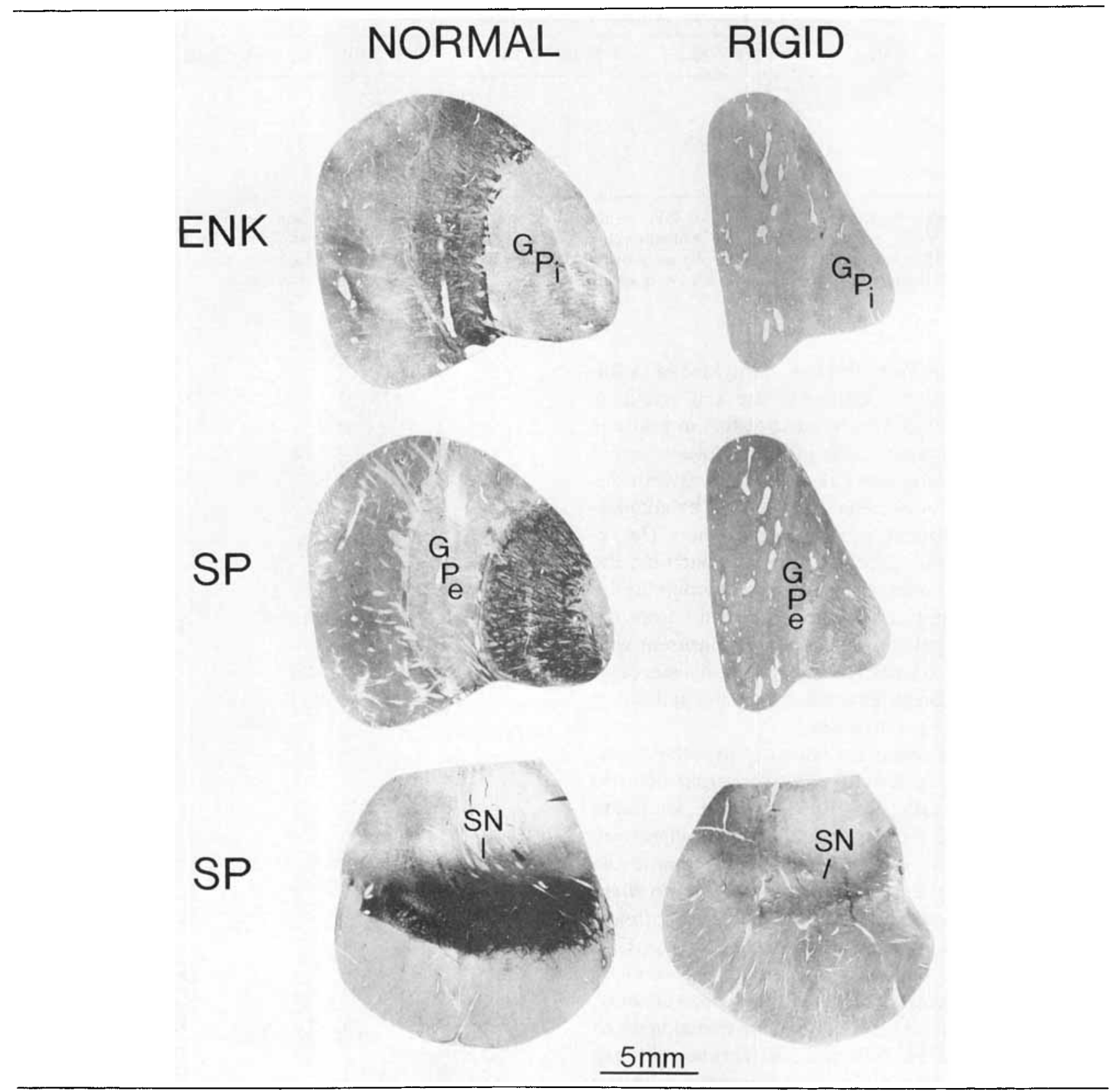

Fig 3. Low-power photomicrographs, comparing enkephalinimmunoreactive (ENK) fiber labeling in external globus pallidus $(G P e)$, substance $P$-immunoreactive (SP) fiber labeling in internal globus pallidus (GPi), and substance $P$-immunoreactive fiber labeling in substantia nigra (SN) in a normal individual with that observed in a brain specimen of rigid, grade 4 juvenile-onset $H D$ (Case 1). Note the depletion of fiber abundance in $G P e, G P i$, and $S N$ in the HD specimens. Scale bar $=$ $5 \mathrm{~mm}$. early in the course of HD, patients with rigid HD should have a greater degree of neuronal depletion in the striatum than those with choreic HD. This prediction is consistent with the observation that juvenileonset $\mathrm{HD}$, which has an enriched percentage of rigid cases $[27,28]$, has a more rapid and severe degenerative course [29]. A recent positron emission tomography (PET) study of patients with juvenile-onset HD showed that they had a greater degree of caudate hypometabolism when compared with patients with adult-onset HD of similar functional capacity [30]; this suggests that juvenile-onset $\mathrm{HD}$ involves a greater degree of striatal cell loss early in the course of the disease than typical adult-onset HD. 

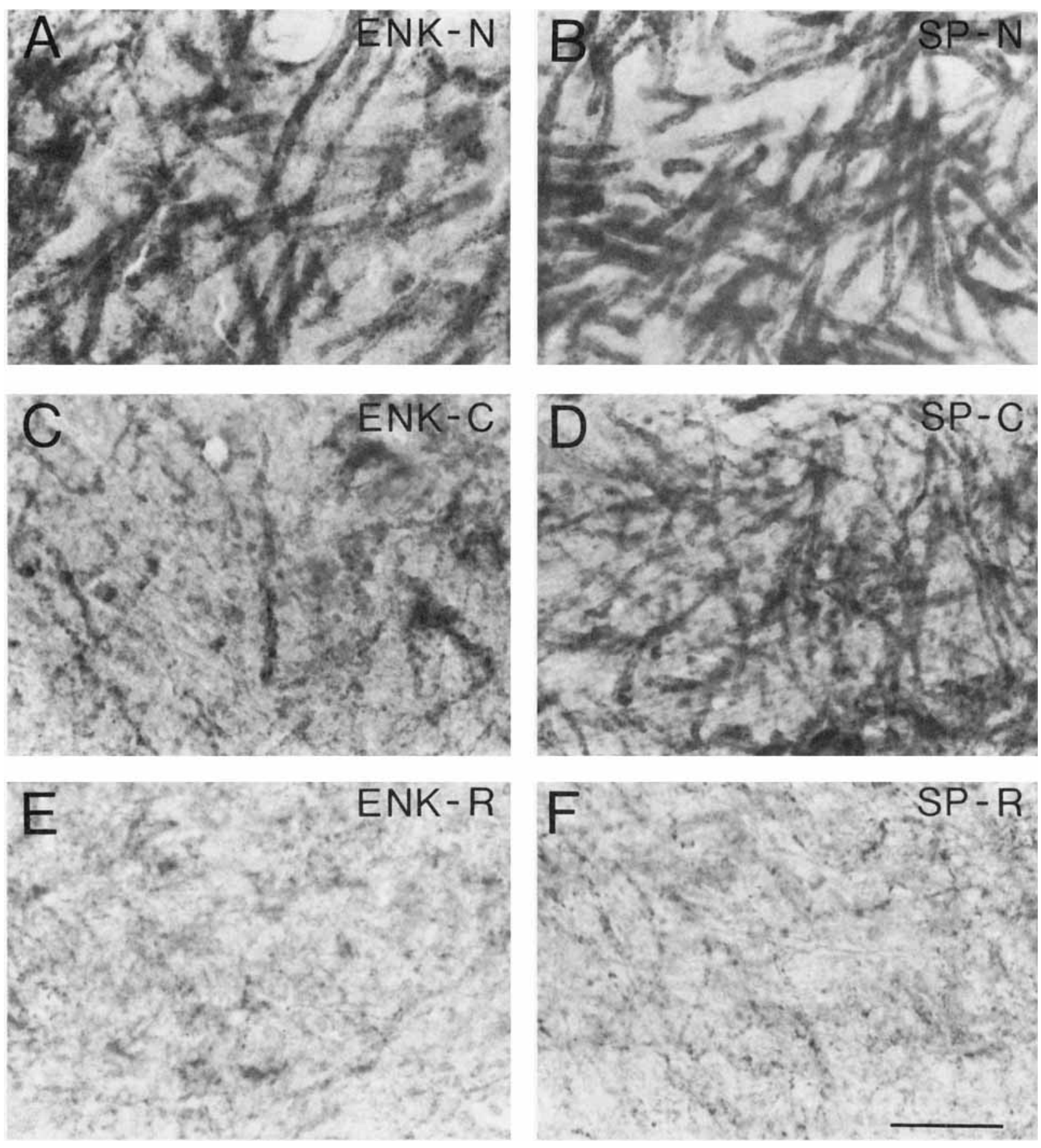

Fig 4. High-power photomicrographs comparing enkephalinimmunoreactive (ENK) fiber labeling in external globus pallidus (A) and substance P-immunoreactive (SP) fiber labeling in internal globus pallidus $(B)$ in a normal juvenile to that observed $(C$ and $D)$ in a case of choreic juvenile-onset $H D$ (grade 3) and to that observed $(E$ and $F$ ) in a case of rigid juvenile-onset $H D$ (Case 3, grade 4). Note the relative preservation of substance $P$ fibers in the internal globus pallidus of the choreic HD specimen, while both substance $P$ and enkephalin fibers in the internal globus pallidus and external globus pallidus, respectively, are largely absent in the case of rigid HD. Scale bar $=50 \mu \mathrm{m}$. $N=$ normal; $C=$ choreic $R=$ rigid.

Stoessel and colleagues suggested that the rigidity of juvenile-onset $\mathrm{HD}$ might be secondary to deficient nigrostriatal dopaminergic neurotransmission [15]. Our finding of normal tyrosine hydroxylase immunoreactivity in the $\mathrm{SN}$ in these brain specimens of rigid $\mathrm{HD}$ indicates that there is no great loss of nigrostriatal projection neurons. Immunohistochemistry cannot assess functional activity and our results do not exclude an abnormality of dopamine metabolism in the striatum, perhaps due to loss of striatal afferents to SN. Spokes, 

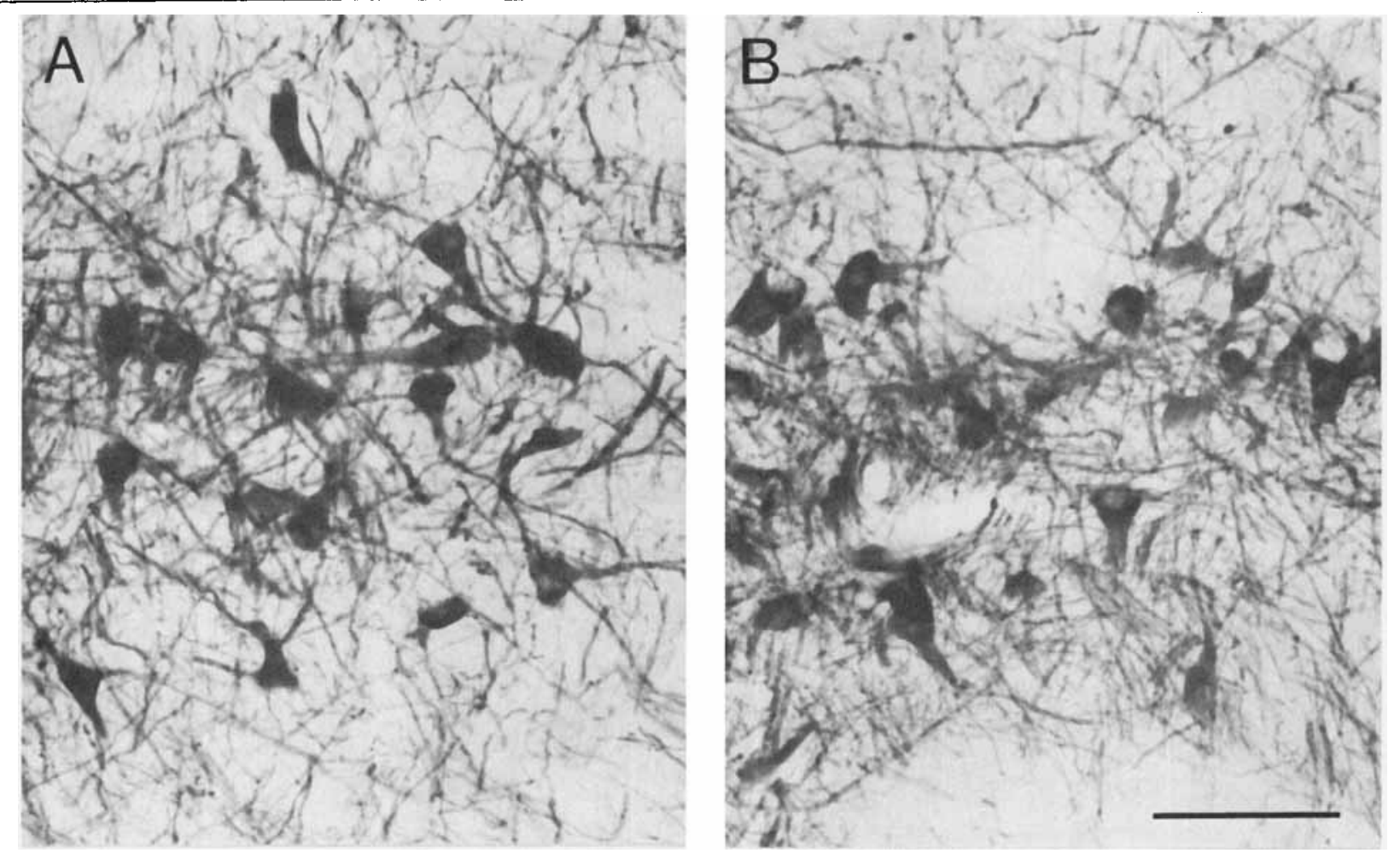

Fig 5. High-power photomicrographs showing that the abundance of tyrosine bydroxylase-immunoreactive perikarya in the substantia nigra of a brain specimen of choreic $H D(A)$ is no different than that in a specimen of rigid $H D(B)$; (Case 3, grade 4). Neither is distinguishable from normal. Scale bar $=$ $200 \mu \mathrm{m}$.

however, showed that there is no difference in striatal dopamine concentrations between patients with rigid HD and those with choreic HD [31], and Kurlan and colleagues showed normal levels of the dopamine metabolite homovanillic acid in the cerebrospinal fluid of patients who had rigid-akinetic HD [32]. These findings from neurochemical studies, coupled with our immunohistochemical data, indicate that nigrostriatal dopaminergic neurotransmission is probably normal in rigid $\mathrm{HD}$.

In conclusion, our findings indicate that the elevated striatal concentration of SS resulting from the persistence in HD of interneurons containing both SS and NPY is unlikely to be the cause of chorea in patients with HD. Furthermore, since tyrosine hydroxylaseimmunoreactive neurons are apparently normal in number in patients with rigid-akinetic HD, it is unlikely that a dopamine deficiency accounts for the rigidakinetic syndrome seen in these patients. Our findings in these brain specimens of rigid-akinetic HD are consistent with the hypothesis that chorea in HD results from selective loss of striatal neurons projecting to the GPe, and that rigidity results from loss of striatal neurons projecting to the GPe and those projecting to

the GPi. Whether rigidity-akinesia would result from selective loss of striatal neurons projecting only to the GPi is an attractive but untested hypothesis.

This work was supported by National Institutes of Health grants NS 19620, NS 19613, NS 15655, and NS 010300; the Hereditary Diseases Foundation; and the Huntington's Disease Society of America.

We thank Debora Romeo for technical assistance. We are indebted to Dr Edward Bird and the staff of the Brain Tissue Resources Center for the provision of HD specimens.

\section{References}

1. Crossman AR. Primate models of dyskinesia: the experimental approach to the study of basal ganglia-related involuntary movement disorders. Neuroscience 1987;21:1-40

2. Villablanca JR, Marcus RJ, Olmstead CE. Effects of caudate nuclei or frontal cortical ablations in cat: I. Neurology and gross behavior. Exp Neurol 1976;52:389-420

3. Kinnier Wilson SA. An experimental research into the anatomy and physiology of the corpus striatum. Brain 1914;36:425-492

4. Shoulson I. On chorea. Clin Neuropharmacol 1986;9:S85-S99

5. Padberg GW, Bruyn GW. Chorea: differential diagnosis. In: Vinken PJ, Bruyn GW, Klawans HL, eds. Handbook of clinical neurology: extrapyramidal disorders. Revised Series 5. New York: Elsevier, 1986:549-564

6. Martin JB, Gusella JF. Huntington's disease. Pathogenesis and management. N Engl J Med 1986;315:1267-1276

7. Penney JB, Young AB. Striatal inhomogeneities and basal ganglia function. Movement Disorders 1986;1:3-15

8. Ferrante RJ, Kowall NW, Beal MF, et al. Selective sparing of a class of striatal neurons in Huntington's disease. Science 1985; 230:561-564 
9. Dawbarn D, Dequidt ME, Emson PC. Survival of basal ganglia neuropeptide Y-somatostatin neurones in Huntington's disease. Brain Res 1985;340:251-260

10. Chesselet M-F, Reisine TD. Somatostatin regulates dopamine release in rat striatal slices and cat caudate nuclei. J Neurosci 1983;3:232-236

11. Beal MF, Martin JB. The effect of somatostatin on striatal catecholamines. Neurosci Lett 1984;44:271-276

12. Fink JS, Martin JB. Behavioral effects of intrastriatal infusions of somatostatin and somatostatin analogues. Soc Neurosci Abstr 1984;10:174

13. Reiner A, Albin RL, Anderson KD, et al. Differential loss of striatal projection neurons in Huntington disease. Proc Natl Acad Sci USA 1988;85:5733-5737

14. Crossman AR, Mitchell JJ, Sambrook MA, et al. Chorea and myoclonus in the monkey induced by gamma-aminobutyric acid antagonism in the lentiform complex. The site of drug action and a hypothesis for the neural mechanisms of chorea. Brain 1988;111:1211-1233

15. Stoessel AJ, Martin WRW, Hayden MR, et al. Dopamine in Huntington's disease: studies using positron emission tomog. raphy. Neurology 1986;36(suppl 1):310(Abstract)

16. Vonsattel JP, Myers RH, Stevens TJ, et al. Neuropathological classification of Huntington's disease. J Neuropathol Exp Neurol 1985;44:559-577

17. Vincent SR, McIntosh CHS, Buchan AMJ, Brown J. Central somatostatin systems revealed with monoclonal antibodies. J Comp Neurol 1985;238:169-186

18. Allen YS, Adrian TE, Allen JM, et al. Neuropeptide Y distribution in rat brain. Science 1983;221:877-879

19. Blessing WW, Howe PRC, Joh TH, et al. Distribution of tyrosine hydroxylase and neuropeptide Y-like immunoreactive neurons in rabbit medulla oblongata, with attention to colocalization studies, presumptive adrenaline-synthesizing perikarya and vagal preganglionic cells. J Comp Neurol 1986;248: $385-400$

20. Beckstead RM, Cruz CJ. Striatal axons to the globus pallidus, entopeduncular nucleus and substantia nigra come mainly from separate cell populations in cat. Neuroscience 1986;19:147158

21. Parent A, Bouchard C, Smith Y. The striatopallidal and striatonigral projections: two distinct fiber systems in primate. Brain Res 1984;303:385-390

22. Feger J, Crossman AR. Identification of different subpopulations of neostriatal neurones projecting to globus pallidus or substantia nigra in the monkey: a retrograde fluorescence double-labelling study. Neurosci Lett 1984;49:7-12

23. Graybiel AM. Neuropeptides in the basal ganglia. In: Martin JB, Barchas JD, eds. Neuropeptides in neurologic and psychiatric disease. New York: Raven Press, 1986:135-161

24. Gerfen CR, Young WS III. Distribution of striatonigral and striatopallidal peptidergic neurons in both patch and matrix compartments: an in situ hybridization histochemistry and fluorescent retrograde tracing study. Brain Res 1988;460:161167

25. Shults $C$, Steardo L, Barone $P$, et al. Huntington's disease: effect of cysteamine, a somatostatin-depleting agent. Neurology 1986; 36:1099-1102

26. Leblanc R, Gauthier S, Gauvin M, et al. Neurobehavioral effects of intrathecal somatostatinergic treatment in nonhuman primates. Neurology 1988;38:1887-1890

27. van Dijk JG, van der Velde EA, Roos RAC, Bruyn GW. Juvenile Huntington's disease. Hum Genet 1986;73:235-239

28. Hayden MR. Huntington's chorea. New York: Springer, 1981:77

29. Myers RH, Vonsattel JP, Stevens TJ, et al. Clinical and neuropathologic assessment of Huntington's disease. Neurology 1988;38:341-348

30. Young AB, Penney JB, Markel DS, et al. Glucose metabolism in juvenile Huntington's disease: comparison with adult onset cases. Neurology 1988;38(suppl 1):360 (Abstract)

31. Spokes EGS. Neurochemical alterations in Huntington's disease. Brain 1980;103:179-210

32. Kurlan R, Goldblatt D, Zaczek R, et al. Cerebrospinal fluid homovanillic acid and parkinsonism in Huntington's disease. Ann Neurol 1988;24:282-284 